\title{
Time-angle sensitivity kernels for sound-speed perturbations in a shallow ocean
}

\author{
Florian Aulanier ${ }^{a)}$ and Barbara Nicolas \\ Image-Signal Department (DIS), Gipsa Lab, Grenoble-INP, University of Grenoble, Grenoble, France \\ Philippe Roux \\ ISTerre, OSUG, University of Grenoble, Grenoble, France
}

Jérôme I. Mars

Image-Signal Department (DIS), Gipsa Lab, Grenoble-INP, University of Grenoble, Grenoble, France

(Received 14 November 2012; revised 17 May 2013; accepted 23 May 2013)

\begin{abstract}
Acoustic waves traveling in a shallow-water waveguide produce a set of multiple paths that can be characterized as a geometric approximation by their travel time (TT), direction of arrival (DOA), and direction of departure (DOD). This study introduces the use of the DOA and DOD as additional observables that can be combined to the classical TT to track sound-speed perturbations in an oceanic waveguide. To model the TT, DOA, and DOD variations induced by sound-speed perturbations, the three following steps are used: (1) In the first-order Born approximation, the Fréchet kernel provides a linear link between the signal fluctuations and the sound-speed perturbations; (2) a double-beamforming algorithm is used to transform the signal fluctuations received on two source-receiver arrays in the time, receiver-depth, and source-depth domain into the eigenray equivalent measured in the time, reception-angle and launch angle domain; and finally (3) the TT, DOA, and DOD variations are extracted from the double-beamformed signal variations through a first-order Taylor development. As a result, time-angle sensitivity kernels are defined and used to build a linear relationship between the observable variations and the sound-speed perturbations. This approach is validated with parabolic-equation simulations in a shallow-water ocean context. (C) 2013 Acoustical Society of America. [http://dx.doi.org/10.1121/1.4809650]
\end{abstract}

PACS number(s): 43.30.Pc, 43.60.Fg, 43.60.Pt [SED]

Pages: 88-96

\section{INTRODUCTION}

In the early 1990s, the sensitivity-kernel approach was introduced into geophysics to improve the performance of ray-based tomography, taking into account the effects of finite-frequency diffraction physics. ${ }^{1}$ Compared to the socalled fat-ray approximation, ${ }^{2}$ sensitivity kernels rely on the more physical wavepath concept, which is closely related to Fresnel tomography in optics. ${ }^{3}$ One striking result from the diffraction physics was the paradoxical banana-doughnut shape of the travel-time sensitivity kernel that links the local perturbations of the propagation medium to the receivedsignal fluctuations. ${ }^{4}$ In recent years, sensitivity kernels of different observables have been proposed [e.g., travel time (TT), amplitude, anisotropy, and even polarization for seismic waves in geophysics ${ }^{5}$ and in ocean acoustics $\left.{ }^{6-8}\right]$, and the relationships between adjoint formulation and time-reversal theory ${ }^{9-11}$ have been developed. The use of sensitivity kernels suggests that higher resolution images can be obtained from this improved description of wave propagation physics.

Later, this theory was carried over to ocean acoustics to model the sensitivity of oceanic waveguide point-to-point records in terms of the local sound speed ${ }^{6}{ }^{6}$ density,${ }^{8}$ and surface-wave perturbations. ${ }^{12}$ Although replacing eigenrays

\footnotetext{
a) Author to whom correspondence should be addressed. Electronic mail: Florian.Aulanier@gipsa-lab.grenoble-inp.fr
}

by sensitivity kernels provides better estimations of signal fluctuations, it does not deal with the waveguide interference problem associated with multipath propagation.

In a shallow-water environment, array processing using source and/or receiver arrays is necessary to improve the separation of the different ray paths. ${ }^{13}$ Recently, Roux et al. ${ }^{14,15}$ proposed a double-beamforming (DBF) algorithm that is based on spatial reciprocity, which takes advantage of both receiver and source arrays. DBF consists of transforming the three-dimensional (3D) data space from time, receiver depth, and source depth into a new 3D space that is related to ray propagation, which is described by the beamformed variables: TT, direction of arrival (DOA), and direction of departure (DOD). As a consequence, every acoustic arrival of the multipath propagation is isolated through DBF and matched to an eigenray according to the TT, DOA, and DOD. When applied to ocean data, Roux et al. ${ }^{14}$ showed that the TT, and also the source and receiver angles of the DBF beams, can be followed as a function of dynamic ocean fluctuations, when, for example, internal waves locally perturb the sound-speed profile (see Figs. 8 and 11 of Ref. 14). Following these observations, the goal of the present study is to connect the TT, DOA, and DOD variations to ocean fluctuations through the formulation of the appropriate sensitivity kernels that describe the forward problem.

Indeed, in the context of source-receiver arrays, the sensitivity kernel is no longer built from one point-to-point 
record, but depends on all of the source-receiver impulse responses. Starting from the definition of point-to-point sensitivity kernel in ocean physics, ${ }^{6}$ this article concentrates on the construction of sensitivity kernels for TT, DOA, and DOD in the framework of a shallow-water waveguide in which DBF is performed to identify and isolate every beam from the source-receiver arrays.

This article is divided into four sections. Section II develops the mathematical formulation of the time-angle sensitivity kernels (T-A-SKs) combined with DBF in a shallow-water environment. The spatial representations of the sensitivity kernels for the TT, DOA, and DOD are presented and discussed in Sec. III, as a function of the aperture of the source-receiver arrays. Using a parabolic-equation (PE) code, Sec. IV deals with a set of numerical simulations that compares the timeangle fluctuations measured in shallow water to their theoretical prediction associated with the sensitivity-kernel formulation, in order to validate the T-A-SK approach.

\section{T-A-SKs}

\section{A. Context and problematics}

Considering classical shallow-water waveguides of about $100 \mathrm{~m}$ in depth and $1 \mathrm{~km}$ in length, and band-limited finite frequency signals of about $1-\mathrm{kHz}$ bandwidth centered around a $2-\mathrm{kHz}$ central frequency, it can be assumed that the sound propagates along ray-like paths. In this context, when an acoustic wave is emitted by a source, the multi-path propagation results in the successive arrival of acoustic wavefronts that interfere at the receiver. In theory, every eigenray can then be characterized in terms of its TT, DOA, and DOD. In practice, however, the measurement of these observables or each eigenray requires the use of sub-arrays that are centered around the source and the receiver, combined with array processing like the DBF algorithm.

This section establishes a linear link between local sound-speed perturbations within an oceanic waveguide, $\delta c\left(\boldsymbol{r}^{\prime}\right)$, and their effects on the TT, DOA, and DOD of the acoustic arrivals: $\delta \tau$ (TT variations), $\delta \Theta_{r}$ (DOA variations), and $\delta \Theta_{e}$ (DOD variations), expressed as

$$
\left(\begin{array}{l}
\delta \tau \\
\delta \Theta_{r} \\
\delta \Theta_{e}
\end{array}\right) \simeq \iiint_{V}\left(\begin{array}{l}
K_{\mathrm{TT}}\left(\boldsymbol{r}^{\prime}\right) \\
K_{\mathrm{DOA}}\left(\boldsymbol{r}^{\prime}\right) \\
K_{\mathrm{DOD}}\left(\boldsymbol{r}^{\prime}\right)
\end{array}\right) \delta c\left(\boldsymbol{r}^{\prime}\right) \mathrm{d} V\left(\boldsymbol{r}^{\prime}\right)
$$

where $V$ is the volume of the whole waveguide and $\mathrm{d} V\left(\boldsymbol{r}^{\prime}\right)$ is an elementary volume located in the waveguide at point $\boldsymbol{r}^{\prime}$.

The TT, DOA, and DOD observables of a given eigenray have their associated sensitivity kernels, as kernels $K_{\mathrm{TT}}$, $K_{\mathrm{DOA}}, K_{\mathrm{DOD}}$, respectively. The process leading to their mathematical expression can be decomposed into three steps:

(1) Sound propagation modeling, which is based on the Born approximation, ${ }^{16}$ and which makes the link between the sound-speed perturbations, $\delta c$, and the received-signal fluctuations, $\delta S\left(\omega, z_{r}, z_{e}\right)$.

(2) The signal-space switch, which uses the DBF technique, and which links the received signal fluctuations, $\delta S\left(\omega, z_{r}, z_{e}\right)$, to the double-beamformed signal fluctuations, $\delta s\left(t, \theta_{r}, \theta_{e}\right)$.
(3) The observable extraction, which uses a first-order Taylor development, and which makes the link between the double-beamformed signal fluctuations, $\delta s\left(t, \theta_{r}, \theta_{e}\right)$, and the observable variations, $\left(\delta \tau, \delta \Theta_{r}, \delta \Theta_{e}\right)$.

\section{B. Step 1: The first-order Born's approximation}

Consider two discrete states of the sound-speed distribution:

(1) $c_{0}(\boldsymbol{r})$, the reference state;

(2) and $c_{p}(\boldsymbol{r})=c_{0}(\boldsymbol{r})+\delta c(\boldsymbol{r})$, the perturbed state, where $\delta c(\boldsymbol{r}) \ll c_{0}(\boldsymbol{r})$ is the perturbation of the sound-speed distribution.

The sound propagation between a source point $\boldsymbol{r}_{e}$ and another point of the waveguide $\boldsymbol{r}$ can be described for a harmonic signal at the frequency $\omega$ by the Green's function $G\left(\omega, \boldsymbol{r}, \boldsymbol{r}_{e}\right)$.

The Green's function in the reference state is denoted as $G_{0}$, and the Green's function in the perturbed state is denoted as $G_{p}\left(\omega, \boldsymbol{r}, \boldsymbol{r}_{e}\right)=G_{0}\left(\omega, \boldsymbol{r}, \boldsymbol{r}_{e}\right)+\delta G\left(\omega, \boldsymbol{r}, \boldsymbol{r}_{e}\right)$, with $\delta G(\omega, \boldsymbol{r}$, $\left.\boldsymbol{r}_{e}\right) \ll G_{0}\left(\omega, \boldsymbol{r}, \boldsymbol{r}_{e}\right)$.

It needs to be noticed that $\delta G$ is the whole acoustic-field fluctuation, which contains the first-order and also the higher-order components of the fluctuation. In the following steps [Born approximation, Eq. (2)] the acoustic field perturbation will be approximated by its first-order component, while the notation will remain the same. Since this article aims at obtaining a linear link between the observable variations and the sound-speed perturbation, the different variables will often be merged with their respective first-order approximations.

Using the first-order Born's approximation, ${ }^{3,6}$ the acoustic field fluctuations $\delta G$ appear to be the solution of the Helmholtz equation in the reference state for the source distribution $\left[2 \omega^{2} / c_{0}^{3}(r)\right] G_{0}\left(\omega, \boldsymbol{r}, \boldsymbol{r}_{e}\right) \delta c(\boldsymbol{r})$ and can be expressed, at the first order, as

$$
\begin{aligned}
& \delta G\left(\omega, \boldsymbol{r}, \boldsymbol{r}_{e}\right) \\
& \quad \simeq \iiint_{V} \frac{-2 \omega^{2}}{c_{0}^{3}\left(\boldsymbol{r}^{\prime}\right)} G_{0}\left(\omega, \boldsymbol{r}, \boldsymbol{r}^{\prime}\right) G_{0}\left(\omega, \boldsymbol{r}^{\prime}, \boldsymbol{r}_{e}\right) \delta c\left(\boldsymbol{r}^{\prime}\right) \mathrm{d} V\left(\boldsymbol{r}^{\prime}\right),
\end{aligned}
$$

where $V$ is the volume defined by the waveguide boundaries.

Using the Fourier transform, Eq. (2), which was established for a harmonic punctual source, is extended to the case of band-limited sources. The received signal fluctuations, $\delta S\left(\omega, \boldsymbol{r}, \boldsymbol{r}_{\boldsymbol{e}}\right)$, at a point $\boldsymbol{r}$ of the waveguide is then in the frequency domain

$$
\delta S\left(\omega, \boldsymbol{r}, \boldsymbol{r}_{e}\right) \simeq \iiint_{V} K_{S}\left(\omega, z_{r}, z_{e}, \boldsymbol{r}^{\prime}\right) \delta c\left(\boldsymbol{r}^{\prime}\right) \mathrm{d} V\left(\boldsymbol{r}^{\prime}\right),
$$

where

$$
K_{s}\left(\omega, z_{r}, z_{e}, \boldsymbol{r}^{\prime}\right)=\frac{2 \omega^{2}}{c_{0}^{3}\left(\boldsymbol{r}^{\prime}\right)} G_{0}\left(\omega, \boldsymbol{r}, \boldsymbol{r}^{\prime}\right) G_{0}\left(\omega, \boldsymbol{r}^{\prime}, \boldsymbol{r}_{e}\right) S_{e}(\omega),
$$

is the Fréchet kernel of the received signal in the $\left(\omega, \boldsymbol{r}_{r}, \boldsymbol{r}_{e}\right)$ domain, ${ }^{4}$ and $S_{e}\left(\omega, \boldsymbol{r}_{e}\right)$ is the spectrum of the signal emitted at a point $\boldsymbol{r}_{e}$ of the waveguide. 


\section{Step 2: The signal-space switch}

DBF (Refs. 14 and 15) is used to switch from the frequency, reception-depth, and transmission-depth domain $\left(\omega, z_{r}, z_{e}\right)$ to the time, reception-angle, and launching-angle domain $\left(t, \theta_{r}, \theta_{e}\right)$. After DBF, the acoustic arrivals can be associated more easily with their corresponding acoustic path. Figure 1 shows examples of eigenrays that represent nine possible ray paths between the centers of the source and receiver arrays. The acoustic arrivals that correspond to each one of these paths can be visualized on the iso-surface representation of the 3D-signal envelope shown in Fig. 2). Using the Huygens-Fresnel principle (summation of coherent sources), this allows the fluctuations of the double-beamformed signal $\delta s\left(t, \theta_{r}, \theta_{e}\right)$ to be written as a function of the signal fluctuations recorded with the source-receive array, $\delta S\left(\omega, z_{r}, z_{e}\right)$,

$$
\begin{aligned}
\delta s\left(t, \theta_{r}, \theta_{e}\right)= & \frac{1}{2 \pi} \int_{-\infty}^{+\infty} \sum_{z_{e}=1}^{N_{e}} \sum_{z_{r}=1}^{N_{r}} \delta S\left(\omega, z_{r}, z_{e}\right) \\
& \times e^{j \omega\left(t+T_{z r}\left(\theta_{r}\right)+T_{z e}\left(\theta_{e}\right)\right)} \mathrm{d} \omega,
\end{aligned}
$$

where

$$
T_{z_{r}}=\int_{z_{r 0}}^{z_{r}} \frac{\sin \left(\theta_{r 0}\right)}{c_{0, \mathrm{r}}(z)} \mathrm{d} z
$$

is the time delay that is introduced to focus around the direction $\theta_{r}$ with the receiver array, and

$$
T_{z_{e}}=\int_{z_{e 0}}^{z_{e}} \frac{\sin \left(\theta_{e 0}\right)}{c_{0, \mathrm{e}}(z)} \mathrm{d} z
$$

is the time delay that is introduced to focus around the direction $\theta_{e}$ with the source array. $z_{e}$ and $z_{r}$ are the source and receiver locations, respectively, and $z_{e 0}$ and $z_{r 0}$ are the locations of the reference source and the reference receiver, respectively. $\mathrm{Nr}$ and $\mathrm{Ne}$ are the number of receivers and sources, respectively.

Introducing Eq. (3) into Eq. (4), and considering $S_{e}(\omega$, $\boldsymbol{r}_{e}$ ) to be constant with respect to $\boldsymbol{r}_{e}$ - which means that each source emits the same signal-the double-beamformed signal fluctuations become at the first order

$$
\delta_{s}\left(t, \theta_{r}, \theta_{e}\right) \simeq \iiint_{V} K_{s}\left(t, \theta_{r}, \theta_{e}, \boldsymbol{r}^{\prime}\right) \delta c\left(\boldsymbol{r}^{\prime}\right) \mathrm{d} V\left(\boldsymbol{r}^{\prime}\right),
$$

where $K_{s}\left(t, \theta_{r}, \theta_{e}, \boldsymbol{r}^{\prime}\right)$ is the Fréchet kernel of the signal in the $\left(t, \theta_{r}, \theta_{e}, \boldsymbol{r}^{\prime}\right)$-domain expressed as

$$
\begin{aligned}
& K_{s}\left(t, \theta_{r}, \theta_{e}, \boldsymbol{r}^{\prime}\right) \\
& \quad=\mathcal{F}^{-1}\left\{\frac{-2 \omega^{2}}{c_{0}^{3}\left(\boldsymbol{r}^{\prime}\right)} G_{0}\left(\omega, \theta_{r}, \boldsymbol{r}^{\prime}\right) G_{0}\left(\omega, \boldsymbol{r}^{\prime}, \theta_{e}\right) S_{e}(\omega)\right\},
\end{aligned}
$$

where $\mathcal{F}^{-1}$ is the inverse Fourier transform $G_{0}\left(\omega, \theta_{r}, \boldsymbol{r}^{\prime}\right)$ $=\sum_{z_{r}=1}^{N_{r}} G_{0}\left(\omega, z_{r}, \boldsymbol{r}^{\prime}\right) e^{j \omega\left(T_{z r}\left(\theta_{r}\right)\right)}$ and $G_{0}\left(\omega, \boldsymbol{r}^{\prime}, \theta_{e}\right)=\sum_{z_{e}=1}^{N_{e}} G_{0}(\omega$, $\left.\boldsymbol{r}^{\prime}, z_{e}\right) e^{j \omega\left(T_{z_{e}}\left(\theta_{e}\right)\right)}$.

\section{Step 3: The observable extraction}

The TT, DOA, and DOD variations of each acoustic arrival, $\left(\delta \tau, \delta \Theta_{r}, \delta \Theta_{e}\right)$, must be linked to the 3D signal fluctuations $\delta s\left(t, \theta_{r}, \theta_{e}\right)$ obtained in Eq. (4).
In the context of an oceanic waveguide of about $1.5 \mathrm{~km}$ in length and $50 \mathrm{~m}$ in depth, for signals with about $2 \mathrm{kHz}$ of central frequency and $1 \mathrm{kHz}$ of bandwidth, and for small sound-speed perturbations, the changes in the modal dispersion between the reference and the perturbed states can be neglected. Therefore, for each acoustic arrival, it is assumed that the changes between the reference and the perturbed state is only a 3D shift of the arrival as time, reception angle, and emission angle, without signal shape changes.

In this context, the acoustic-arrival fluctuations can be characterized by the 3D shift of the acoustic-arrival maximum. As $\left(\tau_{0}, \Theta_{r 0}, \Theta_{e 0}\right)$ is maximum of the acoustic arrival in the reference state, and as $\left(\tau_{p}, \Theta_{r p}, \Theta_{e p}\right)$ is the maximum of the acoustic arrival in the perturbed state, the observable variation is expressed as

$$
\left(\delta_{\tau}, \delta \Theta_{r}, \delta \Theta_{e}\right)=\left(\tau_{p}, \Theta_{r p}, \Theta_{e p}\right)-\left(\tau_{0}, \Theta_{r 0}, \Theta_{e 0}\right) .
$$

In the reference state, at the acoustic-arrival maximum, partial derivatives are null.

Therefore

$$
\left\{\begin{array}{l}
\frac{\partial s_{0}\left(\tau_{0}, \Theta_{r 0}, \Theta_{e 0}\right)}{\partial t}=0, \\
\frac{\partial s_{0}\left(\tau_{0}, \Theta_{r 0}, \Theta_{e 0}\right)}{\partial \theta_{r}}=0, \\
\frac{\partial s_{0}\left(\tau_{0}, \Theta_{r 0}, \Theta_{e 0}\right)}{\partial \theta_{e}}=0,
\end{array}\right.
$$

where $s_{0}\left(\tau_{0}, \Theta_{r 0}, \Theta_{e 0}\right)$ is the value of the doublebeamformed $3 \mathrm{D}$ signal taken at the acoustic arrival maximum in the reference state.

In the perturbed state, at the acoustic arrival maximum the partial derivatives are also equal to zero

$$
\left\{\begin{array}{r}
\frac{\partial s_{p}\left(\tau_{p}, \Theta_{r p}, \Theta_{e p}\right)}{\partial t} \\
=\frac{\partial s_{p}\left(\tau_{0}+\delta \tau, \Theta_{r 0}+\delta \Theta_{r}, \Theta_{e 0}+\delta \Theta_{e}\right)}{\partial t}=0 \\
\frac{\partial s_{p}\left(\tau_{p}, \Theta_{r p}, \Theta_{e p}\right)}{\partial \theta_{r}} \\
=\frac{\partial s_{p}\left(\tau_{0}+\delta \tau, \Theta_{r 0}+\delta \Theta_{r}, \Theta_{e 0}+\delta \Theta_{e}\right)}{\partial \theta_{r}}=0 \\
\frac{\partial s_{p}\left(\tau_{p}, \Theta_{r p}, \Theta_{e \mathrm{p}}\right)}{\partial \theta_{e}} \quad \\
=\frac{\partial s_{p}\left(\tau_{0}+\delta \tau, \Theta_{r 0}+\delta \Theta_{r}, \Theta_{e 0}+\delta \Theta_{e}\right)}{\partial \theta_{e}}=0
\end{array}\right.
$$

Considering that the 3D shift is small, i.e., $\left(\delta \tau, \delta \Theta_{r}\right.$, $\left.\delta \Theta_{e}\right) \ll\left(T_{\omega}, L_{\theta r}, L_{\theta e}\right)$-where $T_{\omega}, L_{\theta r}$, and $L_{\theta e}$ are the signal period, the width of the principal reception lobe, and the width of the principal emission lobe, respectively - the system of Eq. (8) can be developed with a Taylor 
approximation for the first order, and written with the matrix formalism, as

$$
\mathbf{H}\left(s_{p}\right)\left(\tau_{0}, \Theta_{r 0}, \Theta_{e 0}\right) *\left(\begin{array}{l}
\delta \tau \\
\delta \Theta_{r} \\
\delta \Theta_{e}
\end{array}\right)+\mathrm{d}\left(s_{p}\right)\left(\tau_{0}, \Theta_{r 0}, \Theta_{e 0}\right)=0,
$$

where

$$
\mathrm{d}\left(s_{p}\right)\left(\tau_{0}, \Theta_{r 0}, \Theta_{e 0}\right)=\left(\begin{array}{l}
\frac{\partial_{s_{p}}\left(\tau_{0}, \Theta_{r 0}, \Theta_{e 0}\right)}{\partial t} \\
\frac{\partial_{s_{p}}\left(\tau_{0}, \Theta_{r 0}, \Theta_{e 0}\right)}{\partial \theta_{r}} \\
\frac{\partial_{s_{p}}\left(\tau_{0}, \Theta_{r 0}, \Theta_{e 0}\right)}{\partial \theta_{e}}
\end{array}\right),
$$

is the gradient vector of $s_{p}$, taken at $\left(t, \theta_{r}, \theta_{e}\right)=\left(\tau_{0}\right.$, $\left.\Theta_{r 0}, \Theta_{e 0}\right)$; and

$$
\mathbf{H}\left(s_{p}\right)\left(\tau_{0}, \Theta_{r 0}, \Theta_{e 0}\right)=\left(\begin{array}{lll}
\frac{\partial^{2} s_{p}\left(\tau_{0}, \Theta_{r 0}, \Theta_{e 0}\right)}{\partial t^{2}} & \frac{\partial^{2} s_{p}\left(\tau_{0}, \Theta_{r 0}, \Theta_{e 0}\right)}{\partial t \partial \theta_{r}} & \frac{\partial^{2} s_{p}\left(\tau_{0}, \Theta_{r 0}, \Theta_{e 0}\right)}{\partial t \partial \theta_{e}} \\
\frac{\partial^{2} s_{p}\left(\tau_{0}, \Theta_{r 0}, \Theta_{e 0}\right)}{\partial \theta_{r} \partial t} & \frac{\partial^{2} s_{p}\left(\tau_{0}, \Theta_{r 0}, \Theta_{e 0}\right)}{\partial \theta_{r}^{2}} & \frac{\partial^{2} s_{p}\left(\tau_{0}, \Theta_{r 0}, \Theta_{e 0}\right)}{\partial \theta_{r} \partial \theta_{e}} \\
\frac{\partial^{2} s_{p}\left(\tau_{0}, \Theta_{r 0}, \Theta_{e 0}\right)}{\partial \theta_{e} \partial t} & \frac{\partial^{2} s_{p}\left(\tau_{0}, \Theta_{r 0}, \Theta_{e 0}\right)}{\partial \theta_{e} \partial \theta_{r}} & \frac{\partial^{2} s_{p}\left(\tau_{0}, \Theta_{r 0}, \Theta_{e 0}\right)}{\partial \theta_{e}^{2}}
\end{array}\right)
$$

is the Hessian matrix of $s_{p}$ taken at $\left(t, \theta_{r}, \theta_{e}\right)$ $=\left(\tau_{0}, \Theta_{r 0}, \Theta_{e 0}\right)$.

Replacing $s_{p}$ by $s_{0}+\delta s$, the system of equations simplifies through the application of Eq. (7). Then, using the fact that $\delta s\left(t, \theta_{r}, \theta_{e}\right), \mathbf{d}(\delta s)\left(t, \theta_{r}, \theta_{e}\right)$, and $\mathbf{H}(\delta s)\left(t, \theta_{r}, \theta_{e}\right)$ are negligible toward, respectively, $s_{0}\left(t, \theta_{r}, \theta_{e}\right), \mathbf{d}\left(s_{0}\right)\left(t, \theta_{r}\right.$, $\left.\theta_{e}\right)$, and $\mathbf{H}\left(s_{0}\right)\left(t, \theta_{r}, \theta_{e}\right)$; the solution of the matrix system at the first order is written as

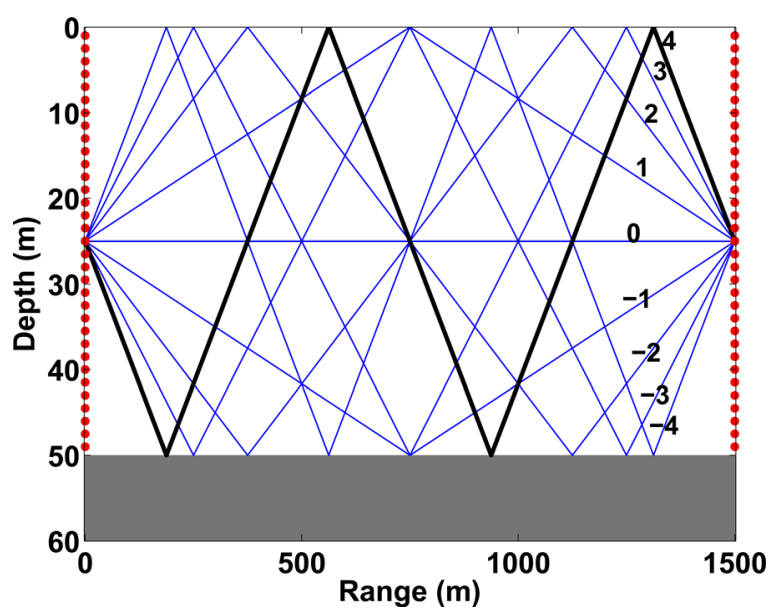

FIG. 1. (Color online) Pekeris waveguide used for the PE simulation. Seawater properties: Sound speed $c_{0}=1500 \mathrm{~m} / \mathrm{s}$; density $\rho_{0}=1000 \mathrm{~kg} / \mathrm{m}^{2}$; and attenuation $\alpha_{0}=0 \mathrm{~dB} / \lambda$. Seabed properties: Sound speed $c_{b}=1600 \mathrm{~m} / \mathrm{s}$; density $\rho_{b}=1600 \mathrm{~kg} / \mathrm{m}^{2}$; and attenuation $\alpha_{b}=0.1 \mathrm{~dB} / \lambda$. The elements of the 48 - $\mathrm{m}$ source-receiver arrays are represented by dots. The acoustic arrivals are indexed by the number of reflections of the corresponding acoustic path. The sign of the reception angle gives the sign of the acoustic arrival/path. The bold black line represents acoustic path number 4 , which is further used in Figs. 3-5.

$$
\left(\begin{array}{l}
\delta \tau \\
\delta \Theta_{r} \\
\delta \Theta_{e}
\end{array}\right) \simeq-\mathbf{H}\left(s_{0}\right)^{-1}\left(\tau_{0}, \Theta_{r 0}, \Theta_{e 0}\right) \cdot \mathrm{d}(\delta s)\left(\tau_{0}, \Theta_{r 0}, \Theta_{e 0}\right),
$$

where it should be recalled that $\delta s$ stands for its first-order approximation as described by Eq. (5).

Once these three steps are done, their outcomes just need to be combined to build the T-A-SK.

\section{E. T-A-SK}

Introducing Eq. (5) into Eq. (12) gives the expected relationship of Eq. (1), which links the sound-speed perturbations to the TT, DOA, and DOD variations by way of the respective sensitivity kernels, $K_{\mathrm{TT}}, K_{\mathrm{DOA}}$, and $K_{\mathrm{DOD}}$, defined as

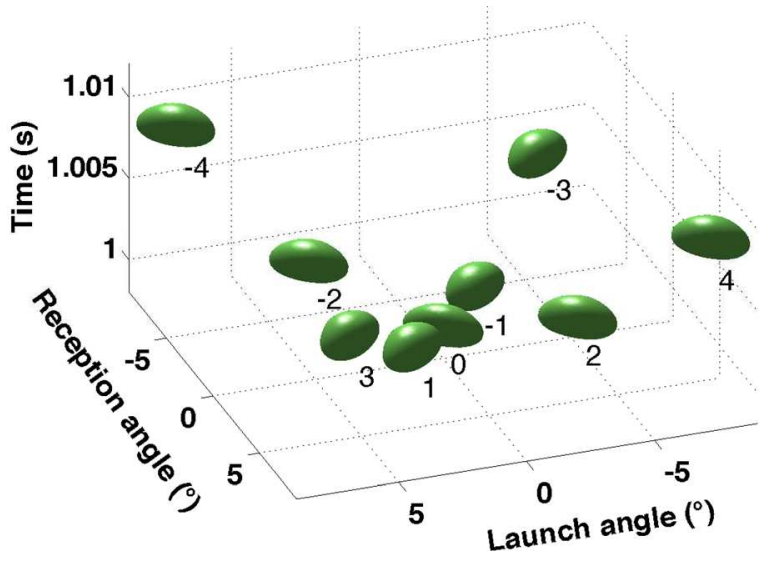

FIG. 2. (Color online) Iso-surface $\left(3.33 \times 10^{-4} \mathrm{~Pa}\right)$ of the $3 \mathrm{D}$-received signal envelope in the double-beamformed domain $\left(t, \theta_{r}, \theta_{e}\right)$. The signal was recorded in the configuration shown in Fig. 1 and the nine "bubbles" represent the nine acoustic arrivals that correspond to the eigenray that is also shown in Fig. 1. 


$$
\begin{aligned}
\left(\begin{array}{c}
K_{\mathrm{TT}}\left(\tau_{0}, \Theta_{r 0}, \Theta_{e 0}, \boldsymbol{r}^{\prime}\right) \\
K_{\mathrm{DOA}}\left(\tau_{0}, \Theta_{r 0}, \Theta_{e 0}, \boldsymbol{r}^{\prime}\right) \\
K_{\mathrm{DOD}}\left(\tau_{0}, \Theta_{r 0}, \Theta_{e 0}, \boldsymbol{r}^{\prime}\right)
\end{array}\right)= & \mathbf{H}\left(s_{0}\right)^{-1}\left(\tau_{0}, \Theta_{r 0}, \Theta_{e 0}\right) \\
& \times \mathbf{d}\left(K_{s}\right)\left(\tau_{0}, \Theta_{r 0}, \Theta_{e 0}, \boldsymbol{r}^{\prime}\right),
\end{aligned}
$$

with $K_{s}$ as defined in Eq. (6).

This formulation of the T-A-SK only uses the reference state to link observable variations and sound-speed perturbations. Consequently, only the Green's function of the reference medium $G_{0}$ and the coordinates of the doublebeamformed acoustic arrival maximum in the reference state, $\left(\tau_{0}, \Theta_{r 0}, \Theta_{e 0}\right)$, are needed.

It can be noted that each observable sensitivity can be influenced by the sensitivity of the others, depending on the shape of the acoustic arrival. This interdependency lies in the cross terms of the Hessian matrix $\mathbf{H}\left(s_{0}\right)$ [Eq. (11)] which links the three kernels together.

\section{AN EXAMPLE OF T-A-SKS IN A SHALLOW-WATER OCEANIC WAVEGUIDE}

\section{A. Framework}

To create sensitivity kernels, it is necessary to simulate the reference Green's function of the oceanic waveguide, $G_{0}$, and the reference received signal, $s_{0}\left(t, z_{r}, z_{e}\right)$. On this basis, a PE code ${ }^{17,18}$ is used to simulate the acoustic propagation in the reference medium described in Fig. 1.

The emitted signal is a sine pulse of $2.5 \mathrm{kHz}$ central frequency and $1.25 \mathrm{kHz}$ bandwidth. This signal is transmitted with a 48 -m-span vertical line array and received with another similar vertical line array located $1500 \mathrm{~m}$ on. Each array has 32 elements that are evenly spaced by $1.5 \mathrm{~m}$. The example of ray number 4 shown in Fig. 1 (black plain line) is taken, with two reflections on the ocean floor and two reflections on the ocean surface.

\section{B. Spatial complementarity of the T-A-SK high-sensitivity zones}

Figures 3-5 show the TT, the DOA, and the DOD sensitivity kernels (TT-SK, DOA-SK, and DOD-SK), respectively, for different apertures of the source-receiver arrays.

The shape of the TT-SKs in Fig. 3 looks like that described by Iturbe et al. ${ }^{7}$ The TT-SKs in Fig. 3(c) are formed of a symmetric and negative sensitivity zone around the ray path, and the sensitivity maximum is on the eigenray. In contrast, the angle sensitivity kernels (A-SK), i.e., the DOA-SKs [Fig. 4(c)] and the DOD-SKs [Fig. 5(c)], have anti-symmetrical high-sensitivity zones with respect to the ray path, and their sensitivity is null on the eigenray. For instance, let us consider two different positive sound-speed perturbations: Perturbation 1, above the ray path, and perturbation 2, below the ray path [see Figs. 3(c), 4(c), and 5(c)]. For both perturbations, the TT will be reduced in the same way, as the sensitivity is $-1.10^{-8} \mathrm{~s} /(\mathrm{m} / \mathrm{s}) / \mathrm{m}^{2}$ at these two perturbation locations. However, as the A-SKs are of opposite signs on both sides of the ray path, the DOD variations will be negative for the first perturbation $\left(-1.10^{-5 \circ} /(\mathrm{m} / \mathrm{s}) / \mathrm{m}^{2}\right)$ and positive for the second perturbation $\left(+1.10^{-5 \circ} /(\mathrm{m} / \mathrm{s}) / \mathrm{m}^{2}\right)$. The same is seen for the DOA, which variates by $-3.10^{-5 \circ} /(\mathrm{m} / \mathrm{s}) / \mathrm{m}^{2}$ for the first perturbation and $+3 \cdot 10^{-5 \circ} /(\mathrm{m} / \mathrm{s}) / \mathrm{m}^{2}$ for the second perturbation.

Furthermore, the TT-SKs [Fig. 3(c)] have symmetrical behavior in terms of the source/receiver, whereas the A-SKs do not. In other words, the TT-SKs are evenly sensitive to

(a)

\begin{tabular}{ccccccccccc}
-2.5 & -2 & -1.5 & -1 & -0.5 & 0 & 0.5 & 1 & 1.5 & 2 & 2.5 \\
\multicolumn{10}{c}{ TT-SK $\left(\mathrm{s} /(\mathrm{m} / \mathrm{s}) / \mathrm{m}^{2}\right)$} \\
\hline
\end{tabular}

(b)
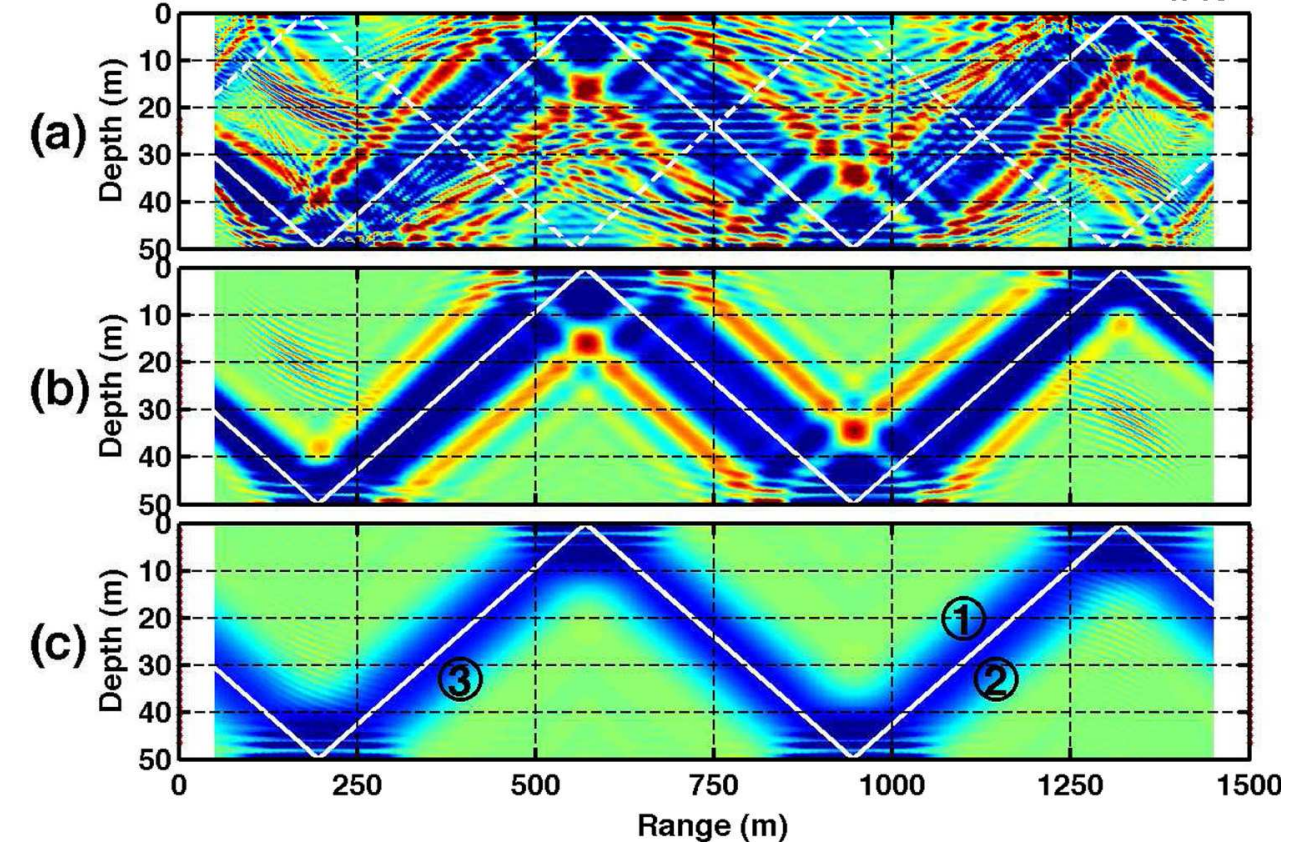

FIG. 3. (Color online) 2D-TT-SKs corresponding to ray path number 4 of Fig. 1 (here a white plain line) for different apertures of the sourcereceiver arrays: (a) 3 , (b) 11 , and (c) 31 elements $(3,15$, and $45 \mathrm{~m}$ span, respectively). The white dashed line shows the ray path with the same TT, but with opposite DOA and DOD. The three numbered circles represent different locations of possible positive perturbations. 
sound-speed perturbations at the beginning and at the end of the waveguide, whereas the DOA-SKs (and the DOD-SKs) are more sensitive to perturbations close to the receiver array (and the source array, respectively), and the DOA sensitivity (and the DOD sensitivity) fades away as it gets farther from the receiver array (and the source array, respectively). Indeed, the Fréchet kernel of the double-beamformed signal [Eq. (6)] is partially derived to obtain the T-A-SKs [Eq. (13)]. The time partial derivative applies in the same way to both $G_{0}\left(\omega, \theta_{r}, \boldsymbol{r}^{\prime}\right)$ and $G_{0}\left(\omega, \boldsymbol{r}^{\prime}, \theta_{e}\right)$, which explains the symmetrical behavior of the TT sensitivity kernel in terms of the emission and reception. Opposite to this, the partial derivative in $\theta_{r}$ applies only to $G_{0}\left(\omega, \theta_{r}, \boldsymbol{r}^{\prime}\right)$ and the partial derivative in $\theta_{e}$ applies only to $G_{0}\left(\omega, \boldsymbol{r}^{\prime}, \theta_{e}\right)$, which implies that the angle sensitivity kernels do not behave symmetrically in terms of the emission or reception.

To illustrate this analysis, consider the examples of the perturbations 2 and 3 [see Figs. 3(c), 4(c), and 5(c)]. Both of these perturbations induce TT variations of $-1.10^{-8} \mathrm{~s} /(\mathrm{m} / \mathrm{s}) / \mathrm{m}^{2}$ despite perturbation 2 being located at the end of the waveguide and perturbation 3 at the beginning [Fig. 3(c)]. Whereas the DOA (and the DOD) variations will be $+3 \cdot 10^{-5 \circ} /(\mathrm{m} / \mathrm{s}) / \mathrm{m}^{2}$ for perturbation 2 [and $+1.10^{-5 \circ} /(\mathrm{m} / \mathrm{s}) / \mathrm{m}^{2}$, respectively) and $+1.10^{-5 \circ} /(\mathrm{m} / \mathrm{s}) / \mathrm{m}^{2}$ for perturbation 3 (and $+3.10^{-5 \circ} /(\mathrm{m} / \mathrm{s}) / \mathrm{m}^{2}$, respectively].

To conclude, for a single ray path, and compared to the information given by the already existing TT-SKs, the A-SKs provide additional spatial information about the location of an eventual sound-speed perturbation that occurs in the waveguide.

\section{Influence of the source-receiver arrays aperture}

Figures 3-5 show the T-A-SKs for three different apertures of the source-receiver arrays, where panels (a), (b), and (c) illustrate the source-receiver arrays of 3, 15, and $45 \mathrm{~m}$, respectively (corresponding to 3,11 , and 31 elements, respectively).

From Figs. 3(a)-3(c), 4(a)-4(c), and 5(a)-5(c), the influence of the array size on the sensitivity kernels can be described according to two effects:

(1) The increase in the source-receiver array aperture allows better separation of the ray path kernels. Indeed, the reference Green's function given by the PE simulation contains all of the possible acoustic arrivals. As point-to-point, it is not possible to separate the sensitivity kernels corresponding to ray paths that are characterized by the same TT. For small source-receiver arrays, the same effect occurs as the angle separation power is weak. This phenomenon can be observed in Figs. 3(a), 4(a), and 5(a), where the sensitivity kernels that correspond to ray paths 4 and -4 (see Fig. 1) overlap with each other. In this case, increasing the source-receiver array aperture up to $9 \mathrm{~m}$ is sufficient to isolate ray path number 4.

(2) High-order Fresnel zones of the sensitivity kernels tend to disappear when the array aperture increases. In the case of the TT-SKs, oscillations give way to a flatter high-sensitivity zone that resembles a fat ray, as described by Iturbe et al. ${ }^{19}$ In the case of the DOA-SKs and DOD-SKs, oscillations give place to two flat highsensitivity zones of opposite sign on either side of the geometrical ray path. The disappearance of the oscillations is accompanied by a diminishing of the sensitivity maximum of the T-A-SKs. These effects can be explained on the basis that after double beamforming, the sensitivity kernels are nothing more than the sum of point-to-point kernels.

(a)

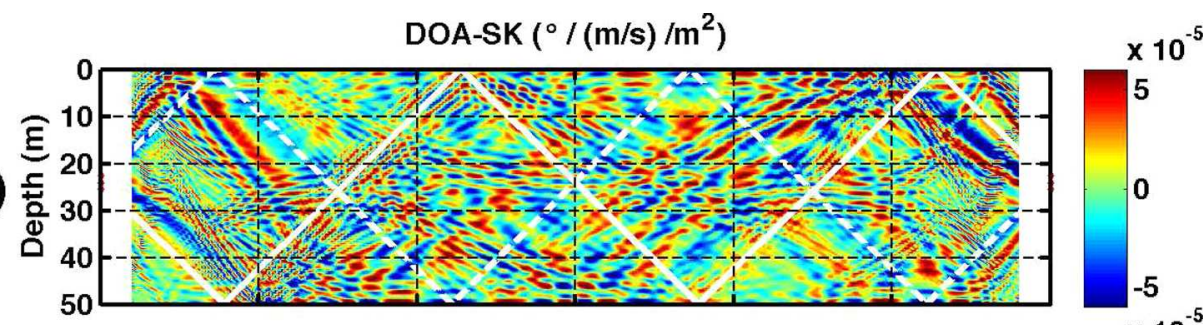

(b)

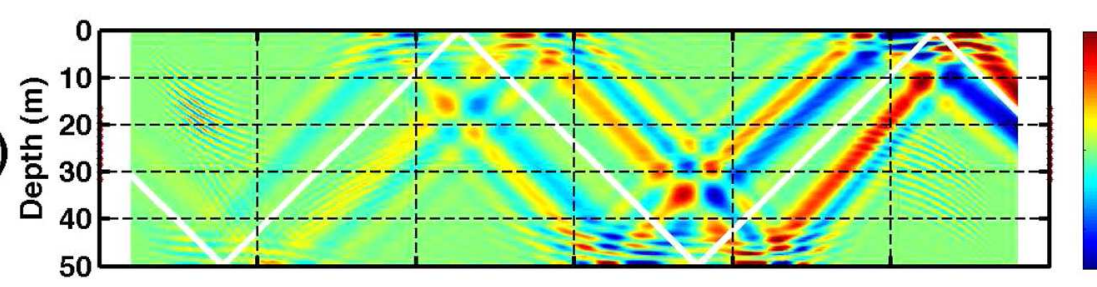
$\times 10^{-5}$

FIG. 4. (Color online) 2D-DOASKs corresponding to ray path number 4 of Fig. 1 (here a white plain line) for different apertures of the source-receiver arrays: (a) 3, (b) 11, and (c) 31 elements $(3,15$, and $45 \mathrm{~m}$ span, respectively). The white dashed line shows the ray path with the same TT, but with opposite DOA and DOD. The three numbered circles represent different locations of possible positive perturbations. 
(a)

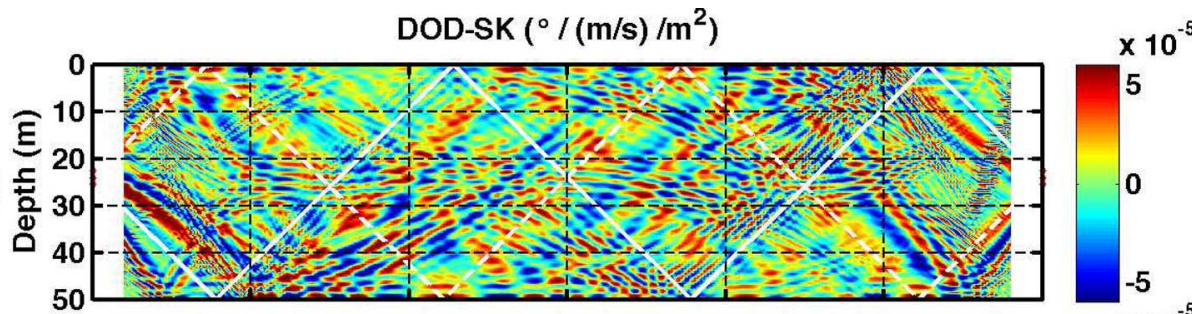

(b)

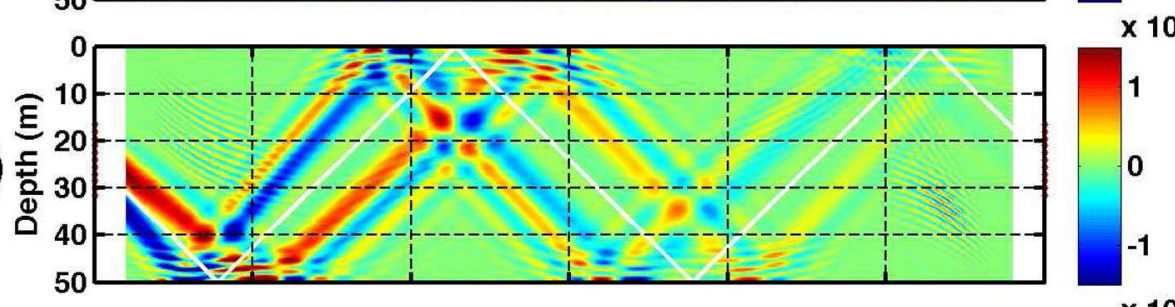

FIG. 5. (Color online) 2D-DODSKs corresponding to ray path number 4 of Fig. 1 (here a white plain line) for different apertures of the source-receiver arrays: (a) 3, (b) 11 , and (c) 31 elements $(3,15$, and $45 \mathrm{~m}$ span, respectively). The white dashed line shows the ray path with the same TT, but with opposite DOA and DOD. The three numbered circles represent different locations of possible positive perturbations.

(c)

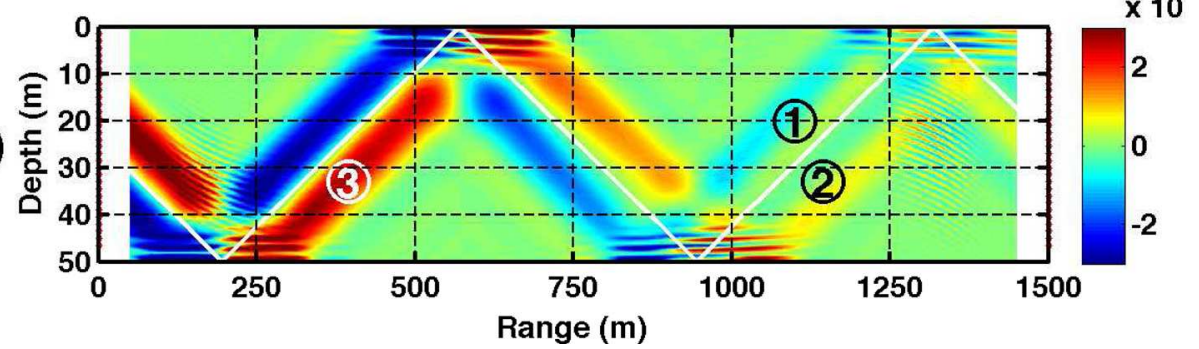

simple deduction. This set of measured observable varia-

\section{T-A-SK VALIDATION USING PE SIMULATIONS}

This section gives the results of some numerical tests that were carried out to validate the T-A-SK approach as a way to estimate the TT, DOA, and DOD variations.

\section{A. Method}

The observable variations [signal-maximum displacement in the $\left(t, \theta_{r}, \theta_{e}\right)$-domain] estimated with the T-A-SKs are compared to the observable variations obtained by measurements on numerical simulations. A two-dimensional (2D)-Padé PE code ${ }^{17,18}$ is used to simulate the sound propagation through the waveguide presented in Fig. 1. The source-receiver arrays used for this acoustic transmission are $21-\mathrm{m}$ arrays of 43 elements that are evenly spaced by $0.5 \mathrm{~m}$. Considering the sound-speed perturbation A [2D-Tukey (tapered cosine) window, as shown in Fig. 6], the outcomes of the PE simulations are:

(a) The Green's functions, $G_{0}\left(\omega, \boldsymbol{r}_{r}, \boldsymbol{r}^{\prime}\right)$ and $G_{0}\left(\omega, \boldsymbol{r}^{\prime}, \boldsymbol{r}_{e}\right)$, for the reference sound-speed distribution $c_{0}(\boldsymbol{r})$;

(b) The Green's function $G_{p}\left(\omega, \boldsymbol{r}_{r}, \boldsymbol{r}_{e}\right)$ for the perturbed sound-speed distribution $c_{p}\left(\boldsymbol{r}^{\prime}\right)$.

The validation process is:

(1) On the one hand, 3D received signals are created for the reference state and the perturbed state. The coordinates of the arrival maximums in the double-beamformed domain (TTs, DOAs, and DODs) are extracted from these 3D signals for the 25 ray paths plotted in Fig. 6 (eigenrays with four reflections on the waveguide boundaries, taken between the five source-subarray-centers and the five receiver-subarray-centers.). For each ray, it is then possible to obtain the measurements of the observable variations between the two states $\left(\delta \tau, \delta \Theta_{e}, \delta \Theta_{e}\right)$ by tions will be used as the "ground truth" for the validation.

(2) On the other hand, the estimates of the observable variations for each of the rays are computed with the T-ASKs.

Figures 7 and 8 summarize these steps. This process is repeated for sound-speed perturbations B, C, and D of Fig. 6 , which provide a set of 100 pairs of measurementestimates for each observable.

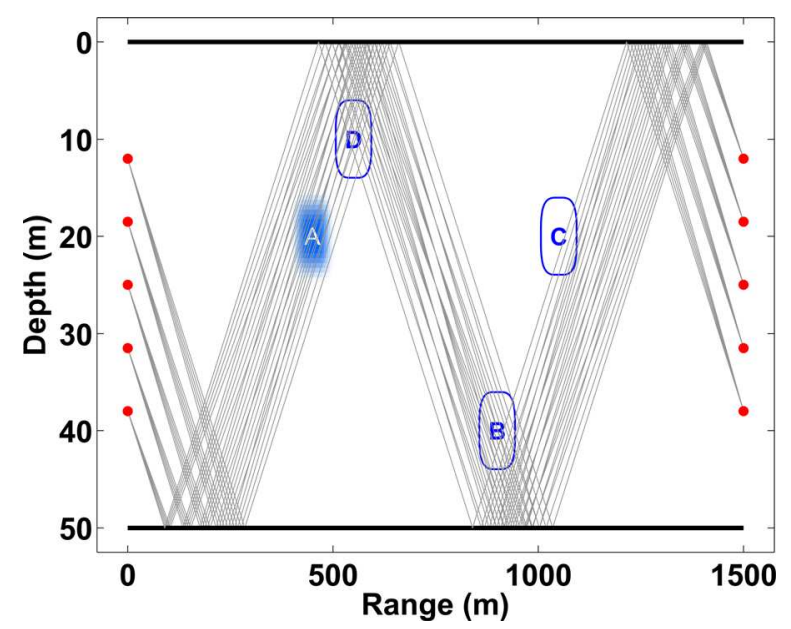

FIG. 6. (Color online) Ray paths used for the T-A-SK validation that correspond to the four-times-reflected eigenrays taken between the center of each subarray (dots). Sound-speed perturbations are 2D-Tukey (tapered cosine) windows with their maximum values at: $0.3 \mathrm{~m} / \mathrm{s}(0.02 \%$ of the sound-speed background). The perturbation shape is given in the "A" case, as shown. The four circles A, B, C, and D show the four locations of the sound-speed perturbations. The reference sensors of the different source-receiver arrays are shown by the dots. 


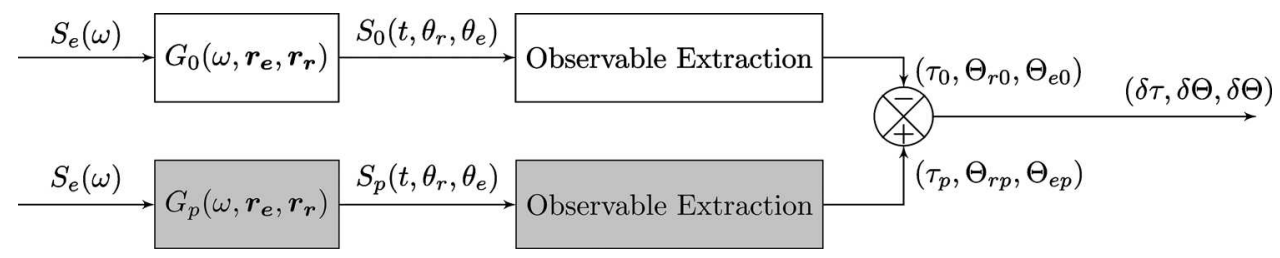

FIG. 7. Flowchart of the main steps of the observable variation measurements carried out with PE simulations. $G_{0}$ and $G_{p}$ are the reference and the perturbed Green's functions, respectively. $S_{0}$ and $S_{p}$ are the 3D signals recorded in the reference and the perturbed states, respectively, and $S_{e}$ is the emitted signal. $\left(\tau_{0}, \Theta_{r 0}, \Theta_{e 0}\right)$ and $\left(\tau_{p}, \Theta_{r p}, \Theta_{e p}\right)$ represent the observables of the acoustic arrival in the reference and perturbed states, respectively. $\left(\delta \tau, \delta \Theta_{r}\right.$, $\left.\Theta_{e}\right)$ are the observable variations measured on the PE-simulated signals shown in the figure.

\section{B. Result analysis}

The results are presented in Fig. 9. This illustrates the measurements of the TT, DOA, and DOD variations carried out on the PE simulations (Fig. 9, circles) and the T-A-SK estimates of the same observable variations (Fig. 9, dots). These variations were observed when the waveguide sound speed distribution was perturbed with the sound-speed perturbations A, B, C, and D (see Fig. 6), successively. At first glance, the T-A-SK estimates almost perfectly match the PE measurements for the TT as well as for the DOA and DOD. Furthermore, the estimates provide a relatively good match of the measurements for several locations of the perturbation inside the waveguide and for several positions of the perturbation with respect to the corresponding ray path. Even for the cases where the perturbations B and D are located relatively close to the interfaces (surface or seafloor) and close to acoustic path rebounds, relatively good results are obtained. With a closer look, it is possible to retrieve some of the T-A-SK features. For instance, perturbation $\mathrm{C}$ perturbs the TTs, DOAs, and DODs of almost all of the acoustic paths, even if only seven of them pass through the perturbation. The finite frequency behavior described in geophysics by Marquering et al. ${ }^{4}$ is retrieved here. Then, it can be seen that the angle variations tend to be positive as well as negative, whereas the TT variations are negative most of the time. The non-symmetrical behavior in terms of the emission/reception is also retrieved here: When perturbed by the sound-speed perturbations A and C, the TTs have the same range of variation, whereas the DOAs are more perturbed by $\mathrm{C}$ than $\mathrm{A}$, and the DODs more by A than $\mathrm{C}$ (see Fig. 9).

Therefore, in the case of perturbation A, it is possible to roughly guess the location of this perturbation by only looking at the observable variations and the T-A-SKs. When the DODs are more perturbed than the DOAs, the perturbation should be closer to the emission, and when almost all of the TTs are perturbed a lot, the perturbation should be on or close to the acoustic paths. Also, as approximately half of the DOAs and DODs are positive and half are negative, perturbation A should be located in the middle of the acoustic paths.

With an even closer look, the T-A-SK estimate errors can be analyzed. Although the sources of this error are not fully identified, some hypotheses can be considered. First, the acoustic path separation is not perfect. Therefore, there remains some interference between the acoustic arrivals that might be a problem for the observable extraction in the PE simulations. Another hypothesis might relate to the way that the PE Green's functions are used. In the flowchart that represents the estimation process with T-A-SK (Fig. 8), there is a product between two Green's functions, $G_{0}\left(\omega, \boldsymbol{r}_{e}, \boldsymbol{r}^{\prime}\right)$ and $G_{0}\left(\omega, \boldsymbol{r}^{\prime}, \boldsymbol{r}_{r}\right)$, whereas in the flowchart that represents the PE measurement process (Fig. 7), the results of the PE simulations, $\mathrm{G}_{0}\left(\omega, \boldsymbol{r}_{e}, \boldsymbol{r}_{r}\right)$ and $G_{p}\left(\omega, \boldsymbol{r}_{e}\right.$, $\left.\boldsymbol{r}_{r}\right)$ are subtracted to obtain the observable variations. It is known that Padé PE simulations can have small phase errors, following the number of Padé coefficients used for the simulation or because of the solution chosen to solve the PE starting field problem. ${ }^{17,18}$ Therefore, because small TT, DOA, and DOD variations are considered here, the differences between multiplying PE Green's functions or subtracting PE Green's function might explain the marginal differences between the T-A-SK estimates and the PE measurements.

\section{v. CONCLUSIONS}

In the context of shallow-water ocean waveguides, the link between local sound-speed perturbations and the TT, DOA, and DOD variations has been theoretically formulated here through T-A-SKs. An example of the T-A-SKs has been shown and explained. The T-A-SKs has been used to estimate the TT, DOA, and DOD in the case of four different perturbations of the sound-speed distribution. These estimates have been compared to measurements made on PE simulations and the T-A-SK approach has been validated in this way.

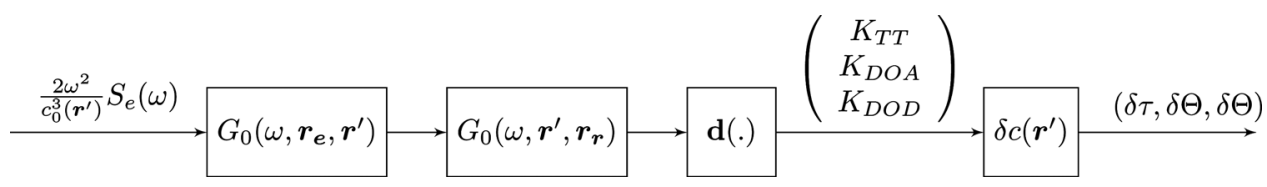

FIG. 8. Flowchart of the observable estimation scheme using the T-A-SKs. Observable variations are induced by a perturbation, $\delta c$, located at a point $\boldsymbol{r}^{\prime}$ in the waveguide. $S_{e}$ is the emitted signal, $G_{0}$ is the reference Green's functions, $\mathbf{d}($.$) is the gradient operator, and \left(\delta \tau, \delta \Theta_{r}, \Theta_{e}\right)$ are the estimates of the observable variations obtained with the T-A-SKs shown in the figure. 
(a)

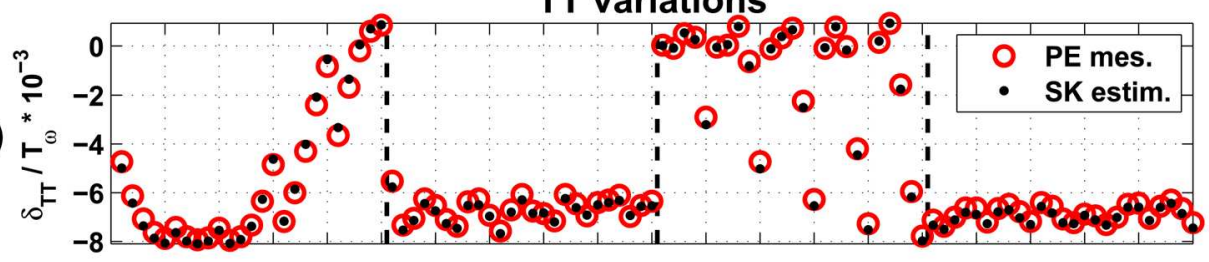

DOA variations

(b)
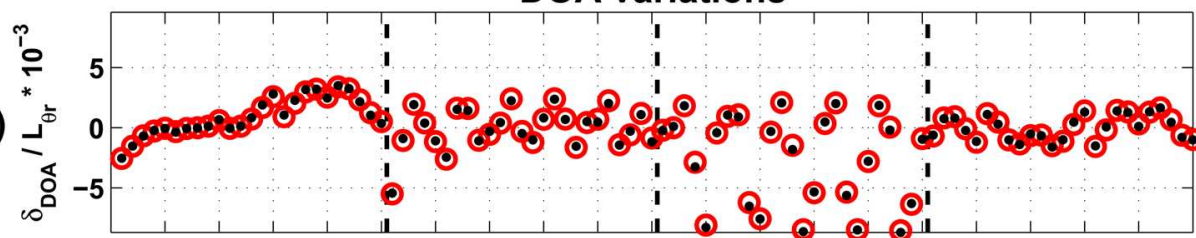

DOD variations

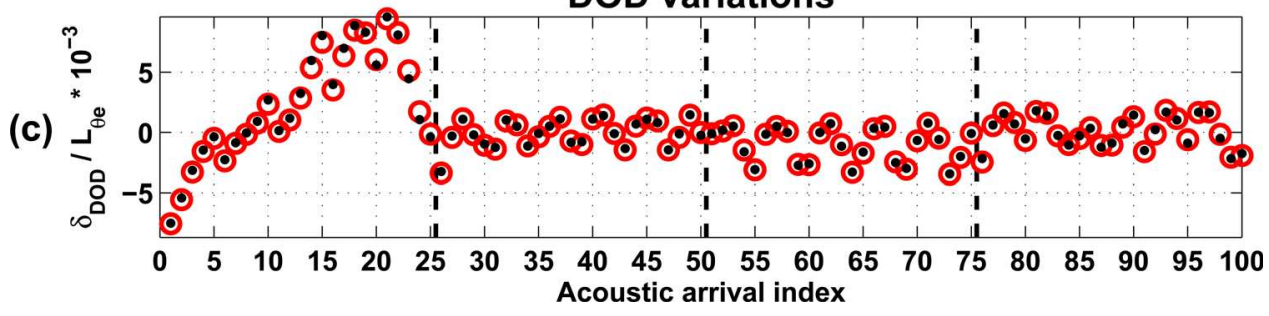

FIG. 9. (Color online) (a) TT variations normalized by the signal period $T_{\omega}=4 \times 10^{-4} \mathrm{~s}$. (b) DOA variations normalized by the main lobe size $L_{\theta r}=2.9^{\circ}$. (c) DOD variations normalized by the main lobe size $L_{\theta e}=2.9^{\circ}$. From left to right, these observable variations are caused by the sound-speed perturbations $\mathrm{A}, \mathrm{B}, \mathrm{C}$, and $\mathrm{D}$ represented in Fig. 6. Circles correspond to measurements made on PE simulations (the ground truth), and dots to the respective T-A-SK estimates.
The angle sensitivity kernels show spatial features that complement those of the time sensitivity kernels. Indeed, DOA- and DOD-SKs show an antisymmetric behavior with respect to the eigen they are related to whereas the TT-SKs are symmetric. At the same time, DOA- and DOD-SKs are more sensitive at the end and the beginning of the waveguide, respectively, whereas the TT-SKs are evenly sensitive.

As the TT, DOA, and DOD variations have been observed and measured in shallow water acoustic data, these results might prove to be promising for ocean acoustic tomography.

\section{ACKNOWLEDGMENTS}

The authors would like to thank Jit Sarkar from the Marine Physical Laboratory, SIO, UC San Diego, for sharing his implementation of the PE code, as well as his programming experience, and Emmanuel Skarsoulis from the Institute of Applied and Computational Mathematics, FORTH, Crete, and Bruce D. Cornuelle, also from the Marine Physical Laboratory, SIO, UC San Diego, for their helpful answers.

${ }^{1}$ M. J. Woodward, "Wave-equation tomography," Proceedings of the 1988 SEG Annual Meeting, Society of Exploration Geophysicists (Anaheim, CA, 1988), pp. 1232-1236.

${ }^{2}$ S. Husen and E. Kissling, "Local earthquake tomography between rays and waves: Fat ray tomography," Phys. Earth Planetary Int. 123, 127-147 (2001).

${ }^{3}$ M. Born and E. Wolf, Principles of Optics: Electromagnetic Theory of Propagation, Interference and Diffraction of Light (Cambridge University Press, Cambridge, UK, 1999), pp. 412-514.

${ }^{4}$ H. Marquering, F. Dahlen, and G. Nolet, "Three-dimensional sensitivity kernels for finite-frequency travel times: The banana-doughnut paradox," Geophys. J. Int. 137, 805-815 (1999).

${ }^{5}$ Y. Zhou, F. Dahlen, and G. Nolet, "Three-dimensional sensitivity kernels for surface wave observables," Geophys. J. Int. 158, 142-168 (2004).
${ }^{6}$ E. K. Skarsoulis and B. D. Cornuelle, "Travel-time sensitivity kernels in ocean acoustic tomography," J. Acoust. Soc. Am. 116, 228-238 (2004).

${ }^{7}$ I. Iturbe, P. Roux, B. Nicolas, J. Virieux, and J. I. Mars, "Shallow-water acoustic tomography performed from a double-beamforming algorithm: Simulation results," IEEE J. Ocean. Eng. 34, 140-149 (2009).

${ }^{8}$ C. Marandet, P. Roux, B. Nicolas, and J. Mars, "Target detection and localization in shallow water: An experimental demonstration of the acoustic barrier problem at the laboratory scale," J. Acoust. Soc. Am. 129, 85-97 (2011).

${ }^{9}$ J. Tromp, C. Tape, and Q. Liu, "Seismic tomography, adjoint methods, time reversal, and banana-doughnut kernels" Geophys. J. Int. 160, 195-216 (2004).

${ }^{10} \mathrm{C}$. Tape, Q. Liu, and J. Tromp, "Finite-frequency tomography using adjoint methods methodology and examples using membrane surface waves," Geophys. J. Int. 168, 1105-1129 (2007).

${ }^{11}$ M. Fink, D. Cassereau, A. Derode, C. Prada, P. Roux, M. Tanter, J. Thomas, and F. Wu, "Time-reversed acoustics," Rep. Prog. Phys. 63, 1933-1995 (2000).

${ }^{12}$ J. Sarkar, C. Marandet, P. Roux, S. Walker, B. Cornuelle, and W. Kuperman, "Sensitivity kernel for surface scattering in a waveguide," J. Acoust. Soc. Am. 131, 111-118 (2012).

${ }^{13}$ L. Jiang, F. Aulanier, G. Le Touzé, B. Nicolas, and J. Mars, "Raypath separation with high resolution processing," IEEE OCEANS 2011 (Spain, 2011), pp. 1-5.

${ }^{14}$ P. Roux, B. D. Cornuelle, W. A. Kuperman, and W. S. Hodgkiss, "The structure of raylike arrivals in a shallow-water waveguide," J. Acoust. Soc. Am. 124, 3430-3439 (2008).

${ }^{15}$ B. Nicolas, I. Iturbe, P. Roux, and J. Mars, "Double formation de voies pour la séparation et l'identification d'ondes: Applications en contexte fortement bruité et à la campagne faf03" ("Wave separation and identification: Applications in high noise level context and to the FAF03 campaign data"), Trait. Signal 25, 293-304 (2008) (in French).

${ }^{16} \mathrm{~W}$. B. Beydoun and A. Tarantola, "First born and Rytov approximations: Modeling and inversion conditions in a canonical example," J. Acoust. Soc. Am. 83, 1045-1055 (1988).

${ }^{17}$ F. Jensen, W. Kuperman, M. Porter, and H. Schmidt, Computational Ocean Acoustics (Springer-Verlag, New York, 2011), pp. 457-527.

${ }^{18}$ M. D. Collins, "A split-step Padé solution for the parabolic equation method,” J. Acoust. Soc. Am. 93, 1736-1742 (1993).

${ }^{19}$ I. Iturbe, P. Roux, J. Virieux, and B. Nicolas, "Travel-time sensitivity kernels versus diffraction patterns obtained through double beam-forming in shallow water," J. Acoust. Soc. Am. 126, 713-720 (2009). 